\section{Typhoid ileal perforation: a 13-year experience}

\author{
Poras Chaudhary, Rajeev Kumar, \\ Chandrakant Munjewar, Utsav Bhadana, \\ Gyan Ranjan, Shailesh Gupta, \\ Sanjay Kumar, Mohinder P. Arora \\ Lady Hardinge Medical College and \\ associated Dr Ram Manohar Lohia \\ Hospital, New Dehli, India
}

\section{Abstract}

Typhoid fever is endemic in many developing countries with a high rate of complications. Aim of this study is to analyse epidemiological features, clinical presentations, complications and therapeutic outcomes of enteric perforation peritonitis diagnosed and treated in our hospital. Records of total number of 646 patients, who presented with perforation peritonitis due to enteric fever in the surgical emergency unit of Dr Ram Manohar Lohia hospital, New Delhi between January 2001 and December 2013, were reviewed retrospectively. Descriptive statistics was used to analyze the data. Out of 646 patients, 62 (9.59\%) presented in shock. Stomal, peristomal, local and systemic complications were high in these patients. Primary closure was done in 212 (33.12) patients, primary ileostomy was created in 410 (64.06) patients, and resection and anastomosis was done in 24 (3.75) patients. Thirteen patients $(2.01 \%)$ died of typhoid intestinal perforation. To prevent complications of typhoid fever, in addition to control sanitation, it is also important to control quackery and malpractices. Awareness and education about the disease, its nature and complications will also be of great help.

\section{Introduction}

Enteric fever is a systemic disease caused by Salmonella typhi and Salmonella paratyphi and it is characterized by fever, abdominal pain, relative bradycardia with involvement of the lymphoid tissues. The serotypes A, B and C cause enteric fever and they have no known hosts other than humans. ${ }^{1}$ The term enteric fever includes both typhoid and paratyphoid fevers. Worldwide, there are an estimated 22 million cases of enteric fever with 200,000 deaths annually. ${ }^{2}$ Typhoid fever is endemic in India. Reported data for the year 2011 shows 1.06 million cases and 346 deaths the prevalence rate of typhoid in India is 88 cases/lac population and death rate due to typhoid is $0.029 /$ lac population. ${ }^{3}$ A high incidence of enteric fever correlates with poor sanitation and lack of access to clean drinking water. The enteric fever has high socio-economic impact because survivors may take several months to recover and resume work. Incubation period is usually 10-14 days but the range may be from 3-56 days depending upon the dose of the bacilli ingested.

Fever is documented at presentation in more than $75 \%$ of the cases and abdominal pain is reported in only $30-40 \%$. Serious complications occur in up to $10 \%$ of patients which depends on host factors like immunosuppression, antacid therapy and vaccination, and strain virulence and inoculum. Life threatening complications, intestinal perforation (1-3\%), gastrointestinal bleeding (10-20\%) and circulatory collapse most commonly occur during third week of illness. In treated cases, the fatality rates of enteric fever range from $1-4 \%$ and in untreated cases, the fatality rates may rise to $10-20 \%$.

The most common surgical complication of enteric fever in India is intestinal perforation, which carries a high morbidity and mortality. This is a retrospective study on our experiences with enteric perforation peritonitis in the past 13 years. The aim of this retrospective study was to analyze epidemiological features, clinical presentations, complications and therapeutic outcomes of enteric perforation peritonitis diagnosed and treated in our hospital.

\section{Materials and Methods}

Records of a total number of 646 patients, who presented with perforation peritonitis due enteric fever in the surgical emergency unit of Dr Ram Manohar Lohia hospital, New Delhi between January 2001 and December 2013, were reviewed retrospectively. Patients with associated malignancy and HIV were excluded from the study.

Records of these patients were then reviewed in detail to analyze epidemiological features, clinical presentations, complications and therapeutic outcomes of enteric perforation peritonitis. The parameters including age, gender, socioeconomic status, complete blood count, liver and kidney function test, serum electrolytes at presentation and after surgery, diagnostic procedures, duration of hospital stay, post-operative morbidity, mortality, medical/surgical treatment and socioeconomic impact were evaluated. Blood culture was done in all of these patients which confirmed enteric fever. Descriptive statistics was used to summarize the data.

\section{Results}

There were 435 male and 211 female patients. Their mean age was 29 (range 14-76)
Correspondence: Poras Chaudhary, Lady Hardinge Medical College and associated Dr Ram Manohar Lohia Hospital, 189 Deoli Road, 110062 New Dehli, India.

Tel.: +91.9891.4473.358.

E-mail: drporaschaudhary@yahoo.com

Key words: Typhoid fever; Intestinal perforation High morbidity; Awareness and education.

Received for publication: 23 August 2014.

Revision received: 6 April 2015.

Accepted for publication: 7 April 2015.

This work is licensed under a Creative Commons Attribution 3.0 License (by-nc 3.0).

(C) Copyright P. Chaudhary et al., 2015

Licensee PAGEPress, Italy

Healthcare in Low-resource Settings 2015; 3:4677 doi:10.4081/hls.2015.4677

(Table 1). Total leucocyte count was found to be high in 598 patients with a mean of $16,500 /$ cu.mm. and less than $4000 /$ cu.mm. in 48 patients only. Renal function tests were deranged in 621 patients and electrolyte imbalance was found in 612 patients. Out of these 646 patients 62 presented in shock. Exploratory laparotomy was possible in 56 patients after resuscitation and drain insertion and 6 patients died before surgery only due septic shock. Out of these 56 patients, 3 patients died after surgery and out of 584 patients without shock at presentation, 8 patients died after surgery due to septic shock and multiple organ dysfunction syndrome.

Stomal, peristomal, local and systemic complications were high in these patients (Table 2). Primary closure was done in 212 patients, primary ileostomy was created in 410 patients and resection and anastomosis was done in 24 patients (Table 3). Reperforations requiring surgery were observed in 43 patients. These perforations occurred proximal to previous perforation site in 39 cases while distal to the first perforation in 4 cases. In all of these cases perforation site was brought out as ileostomy during first surgery.

\section{Discussion}

Typhoid fever occurs in all parts of the world where water supplies and sanitation are substandard. The incidence is highest in southcentral and south-east Asia. Though it is easy to diagnose and cure enteric fever, the complication rate is still very high in developing countries including India. Ileal perforation is the most common surgical complication of enteric fever in India. ${ }^{4}$ One reason is obvious, 
i.e. sub-standard water supplies and sanitation. Other reasons include persistence of quackery in almost every part of the country including metropolitan cities. Typhoid fever is more common in low socioeconomic group, though it occurs in upper and middle class as well, but incidence is comparatively less and complication rate is almost negligible in upper and middle class. This difference of incidence of typhoid fever and its complication is due to the two reasons mentioned previously. People in low socio-economic groups almost always first go to quacks and they misdiagnose and misguide patients, which results in increased complication rates. Out of 646 patients, 412

Table 1. Patients' characteristics.

Sex (M:F)

$435: 211$

Low socioeconomic status (n)

Duration of symptoms before seeking medical advice (days)

Patients presented in shock (n)

Operative time (min)

ICU care

$$
\begin{array}{ll}
\text { Yes } & \text { Immediate after surgery } \\
& \text { Later during post-op period } \\
\text { No } &
\end{array}
$$

9

62

50 (range 45-90)

Patients requiring antibiotics other than IV ceftriaxone (n)

Hospital stay (days)

Patients presented to quacks initially before coming to government centres (n)

Mortality (n)

\begin{tabular}{|c|c|c|c|}
\hline \multirow[t]{2}{*}{ Complications } & & \multicolumn{2}{|c|}{ Patients ( $\mathrm{n}=633$ ) } \\
\hline & & n & $\%$ \\
\hline \multirow{5}{*}{ Stomal and peristomal } & Skin excoriation & 408 & 64.45 \\
\hline & High output & 53 & 8.37 \\
\hline & Retraction & 13 & 2.05 \\
\hline & Prolapse & 87 & 13.74 \\
\hline & Parastomal hernia & 9 & 1.42 \\
\hline \multirow[t]{4}{*}{ Systemic } & Acute renal failure & 16 & 2.52 \\
\hline & ARDS & 18 & 2.84 \\
\hline & Pneumonitis & 76 & 12.01 \\
\hline & Atelectasis & 68 & 10.74 \\
\hline \multirow{8}{*}{ Secondary to primary surgery } & Anastomotic or primary closure site leak & 48 & 7.58 \\
\hline & Intra-abdominal collections & 65 & 10.26 \\
\hline & Wound dehiscence Superficial & 512 & 80.88 \\
\hline & weep & 84 & 13.27 \\
\hline & Prolonged ileus & 92 & 14.53 \\
\hline & Severe electrolyte imbalance & 77 & 12.16 \\
\hline & Adhesive intestinal obstruction & 32 & 5.05 \\
\hline & Incisional hernia & 31 & 4.89 \\
\hline
\end{tabular}

ICU, intensive care unit.

Table 2. Complications of typhoid enteric perforation after surgery.

\begin{tabular}{|c|c|c|}
\hline Type of surgery & & \\
\hline & n & $\%$ \\
\hline Perforation site brought out as stoma $(n=640)$ & 410 & 64.06 \\
\hline Primary closure $(n=640)$ & 212 & 33.12 \\
\hline Resection and anastomosis $(n=640)$ & 24 & 3.75 \\
\hline $\begin{array}{ll} & \text { Reperforation after stoma creation }(n=410) \\
\text { Resurgery for } & \text { Leak after primary closure }(n=212) \\
& \text { Leak after } R \& A(n=24)\end{array}$ & $\begin{array}{l}43 \\
32 \\
16\end{array}$ & $\begin{array}{l}10.48 \\
15.09 \\
66.66\end{array}$ \\
\hline
\end{tabular}

ARDS, acute respiratory distress syndrome.

Table 3. Operative procedures done for typhoid enteric perforation.

R\&A, resection and anastomosis. 
(63.77) initially went to quacks for management of fever for which they received empirical treatment. Patients attend government tertiary care centers after complications arise. Government tertiary care centers are very well equipped and provide free services to all the patients. Poor patients who seek early advice during the course of illness in tertiary care centers almost never develop complications like perforation peritonitis and intestinal haemorrhage.

Ileal perforation peritonitis is the most common surgical complication encountered in our institute. Six hundred forty six patients presented with perforation of ileum in surgical emergency. The authors have studied only those patients who presented in surgical unit 6 , so actual burden of these complications is much more. Out of these 646 patients, 62 presented in shock. Aggressive resuscitation and insertion of abdominal drain before taking up patients for definitive surgery were helpful in saving life. Exploratory laparotomy was possible in 56 patients after resuscitation and drain insertion. Kouame and colleagues ${ }^{5}$ also recommended the importance of aggressive resuscitation before surgery.

Stomal, peristomal and systemic complications are more with enteric perforations as compared to perforation secondary to other causes. ${ }^{4}$ Wound dehiscence, and post-operative intra-abdominal collections including abscess were the most common complications requiring resurgery. Reperforations requiring surgery were observed in 43 patients. These perforations occurred proximal to previous perforation site in 39 cases while distal to the first perforation in 4 cases. In all of these cases perforation site was brought out as ileostomy during first surgery. During resurgery, new perforation site was then brought out as ileostomy and distal part was resected. Three patients developed perforations after second surgery proximal to previous perforation site and during third surgery stoma was refashioned. Reperforations were possibly due to presence of multiple ulcers resulting in perforation at these ulcer sites. Patients who were not diagnosed properly initially and did not receive any treatment for enteric fever resulted in progression of pathology in the form of multiple ulcers and perforation at different times.

Primary closure was done in 212 patients, out of which leak was observed in 32 patients and a stoma was created in these 32 patients during resurgery. Resection and anastomosis was done in 24 patients and leak from anastomotic site was observed in 16 patients and during resurgery double barrel ileostomy was created in 6 patients and in 10 patients, end ileostomy with closure of distal ascending colon loop was done. Zida and colleagues ${ }^{6}$ recommended creation of ileostomy as primary therapy for ileal perforation peritonitis as it reduces morbidity and mortality while $\mathrm{Pal}$ and colleagues $^{7}$ recommended primary closure and side to side ileotransverse for better results.

Surgical site infection (SSI) is one of the major complications in these patients. Superficial incisional surgical site infections, involving only skin and subcutaneous tissue, occurred in 512 patients while deep incisional surgical site infections were seen in 84 patients. All the patients who presented in shock in emergency and then underwent surgery after resuscitation and broad spectrum antibiotic coverage developed deep incisional SSIs. Out of these 84 patients, 69 underwent resurgery. Surgical site infections are more common in patients with shock because shock results in reduction of local perfusion which enhances susceptibility to infection and a little load of organisms is required to produce infection in presence of shock. ${ }^{8}$ Fluid and electrolyte imbalance was seen in all the patients and persisted for a varying period of length after surgery. Fluid and electrolyte imbalance results in inadequate perfusion of gastrointestinal tract and increases chances of SSI. Surgical site infection resulted in impaired mobility, increased hospitalization, delayed rehabilitation and incisional hernia.

Mortality due to complication of this benign disease was high. Out of 62 patients who presented with shock, 6 died even before surgery. Rest of the 56 patients underwent surgery after aggressive resuscitation and drain insertion under local anaesthesia. After surgery, 3 of these patients died due to septic shock and multiple organ dysfunction syndrome. Out of 584 patients, 8 died due to septic shock. The total number of deaths in present study was 13 (2.01\%), while Mogasale and colleagues ${ }^{9}$ reported 706 deaths out of 4626 patients. Atamanalp and colleagues ${ }^{10}$ stressed upon the role of early and appropriate surgical intervention to decrease morbidity and mortality.

\section{Conclusions}

To conclude, treatment of cases, contacts and carriers is important to prevent complications. Typhoid fever and its complications are never a major problem where there is clean water supply and very well established modern public health and these are accomplished fact in most of the developed countries. To prevent complications of typhoid fever, in addition to control sanitation, it is also important to con- trol quackery and malpractices. Awareness and education about the disease, its nature and complications, and about the potential hazards of using contaminated food and water will also be of great help. Moreover, vaccines are available for typhoid, though typhoid vaccination is presently not part of the national immunization programme. Still, vaccination alone cannot control typhoid fever and its complications. This awareness can be spread through national programmes which are still lacking in India.

\section{References}

1. Ananthnarayanan R, Paniker CKJ. Enterobacteriaceae III: salmonella textbook of medical microbiology. 8th ed. Hyderabad; Universities Press; 2009. pp 288-300.

2. Park K. Epidemiology of communicable diseases. Park's textbook of preventive and social medicine. $21^{\text {st }}$ ed. Bhanot; Jabalpur: 2011. pp 213-6.

3. Government of India. National health profile 2011. New Delhi; Ministry of Health and Family Welfare: 2012.

4. Chaudhary P, Nabi I, Ranjan G, et al. Prospective analysis of indications and early complications of emergency temporary loop ileostomies for perforation peritonitis. Ann Gastroent Hepato 2014;27:1-6.

5. Kouame J, Kouadio L, Turguin HT. Typhoid ileal perforation: surgical experience of 64 cases. Acta Chir Belg 2004;104:445-7.

6. Zida M, Ouedraogo T, Bandre E, et al. Primary ileostomy for typhoid-related ileal perforation: a 62-case series in Ouagadougou, Barkina Faso. Med Trop (Mars) 2010;70:267-8.

7. Pal DK. Evaluation of best surgical procedures in typhoid perforation: an experience of 60 cases. Trop Doct 1998;28:16-8.

8. Meakins JL, Masterson BJ. ACS surgery: principles and practice. In: Souba WW, Fink MP, Jurkowich GJ, Kaiser LR, Pearce WH, Pemberton JH, Soper NJ, eds. American College of surgeons. New York, NY: WebMD; 2007. p 27.

9. Mogasale V, Desai SN, Mogasale VV, et al. Case fatality rate and length of hospital stay among patients with typhoid intestinal perforation in developing countries: a systematic literature review. PLoS One 2014;17:e93784.

10. Atamanalp SS, Avdinli B, Ozturk G, et al. Typhoid intestinal perforation: twenty-six year experience. World J Surg 2007;31: $1883-8$. 